\title{
Electronic structure of copper nitrides as a function of nitrogen content
}

\author{
N. Gordillo ， R. Gonzalez-Arrabal ，P. Diaz-Chao ，J.R. Ares ，I.J. Ferrer , \\ F. Yndurain , F. Agulló-López
}

\begin{abstract}
A B S T R A C T
The nitrogen content dependence of the electronic properties for copper nitride thin films with an atomic percentage of nitrogen ranging from $26 \pm 2$ to $33 \pm 2$ have been studied by means of optical (spectroscopic ellipsometry), thermoelectric (Seebeck), and electrical resistivity measurements. The optical spectra are consistent with direct optical transitions corresponding to the stoichiometric semiconductor $\mathrm{Cu}_{3} \mathrm{~N}$ plus a free-carrier contribution, essentially independent of temperature, which can be tuned in accordance with the $\mathrm{N}$-excess. Deviation of the $\mathrm{N}$ content from stoichiometry drives to significant decreases from -5 to $-50 \mu \mathrm{V} / \mathrm{K}$ in the Seebeck coefficient and to large enhancements, from $10^{-3}$ up to $10 \Omega \mathrm{cm}$, in the electrical resistivity. Band structure and density of states calculations have been carried out on the basis of the density functional theory to account for the experimental results.
\end{abstract}

\section{Introduction}

Copper nitrides thin films have attracted much attention in the last decades because of their potential to be employed in nanophotonics and spintronics devices, including permanent optical storage media [1-6]. In spite of the promising properties of $\mathrm{Cu}_{3} \mathrm{~N}$, the large discrepancies reported in literature about its measured physical properties have hampered the implementation of reliable technological devices. As an example, the electric conductivity has been reported to range from a quasi-metallic [7-12] $\left(10^{3} \Omega^{-1} \mathrm{~cm}^{-1}\right)$ to a semiconducting behavior [13-17] $\left(10^{-3} \Omega^{-1} \mathrm{~cm}^{-1}\right)$ and the optical energy gap values cover a wide range [15-19] $(0.9-1.9 \mathrm{eV})$. This dispersion of data could be mostly attributed to differences in the stoichiometry of the films, which in most works has not been appropriately characterized. Indeed, as in other metal nitrides, the stoichiometry is expected to determine the physical properties of the material $[10,20-22]$.

$\mathrm{Cu}_{3} \mathrm{~N}$ is an intrinsic metastable semiconductor presenting an open cubic anti- $\mathrm{ReO}_{3}$ (Fig. 1a) crystal structure [10,15-17,23], which is quite suited for the incorporation of other elements such as $\mathrm{Cu}, \mathrm{N}, \mathrm{Ag}$ and Pd in the interstices of the unit cell. Although some information is available $[11,24]$ on the effect of $\mathrm{Cu}$-doping on $\mathrm{Cu}_{3} \mathrm{~N}$, the situation concerning $\mathrm{N}$-doping is less clear. Density functional theory calculations have indicated that the addition of $\mathrm{N}$ extra atoms to form $\mathrm{Cu}_{3} \mathrm{~N}_{2}$, introduces some energy bands near the Fermi level, modifying the $\mathrm{Cu}_{3} \mathrm{~N}$ electronic structure and leading to a semiconductor to metal transition [25]. Unfortunately, these theoretical predictions have not been, corroborated by experiments, very probably because of the instability of the $\mathrm{Cu}_{3} \mathrm{~N}_{2}$ compounds, which as far as we know have not been hitherto synthesized. An extensive research effort $[10,26,27]$ has been recently devoted to understand the structure and physical properties of $\mathrm{N}$-rich films, whose stoichiometry has been well characterized by means of ion beam analysis techniques. A main problem that has been addressed concerns the location of the excess $\mathrm{N}$ atoms, that has been, so far, a matter of controversy. Experimental data [27] have revealed that for films sputtered under the same conditions, extra nitrogen (or at least a high fraction of it) incorporates in the film in interstitial positions forming a solid solution and expanding the crystal lattice in comparison to stoichiometric $\mathrm{Cu}_{3} \mathrm{~N}$. Moreover, spectroscopic ellipsometry and infrared absorption measurements carried out on $\mathrm{N}$-rich $\mathrm{Cu}_{3} \mathrm{~N}$ films have clearly indicated the presence of a free-carrier contribution in the optical spectra that has been related to the $\mathrm{N}$ excess [26]. The origin and nature of those extra carriers could not be elucidated in that work, partly due to the difficulty of carrying out reliable Hall measurements and the dispersion of so far reported data $[23,28]$.

In order to clarify the experimental situation and to assess the electronic role of the excess $\mathrm{N}$ in $\mathrm{Cu}_{3} \mathrm{~N}$ experiments: electrical conductivity, spectroscopic ellipsometry at several temperatures and 
a)

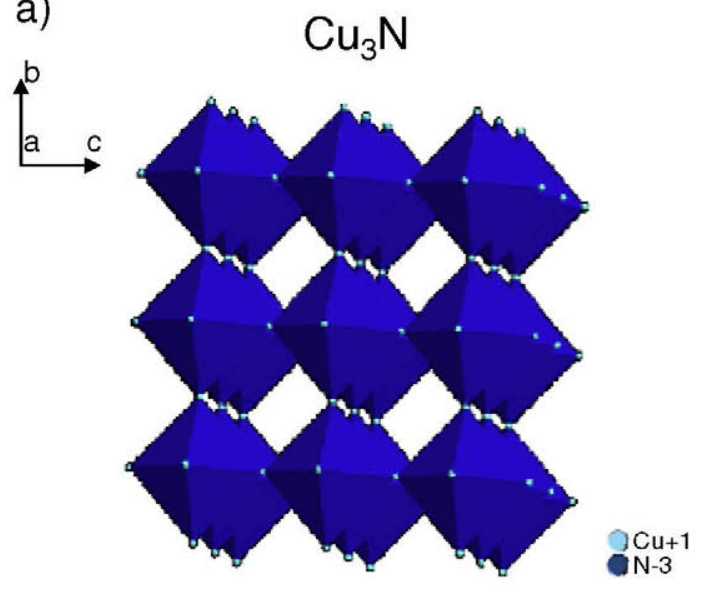

b)

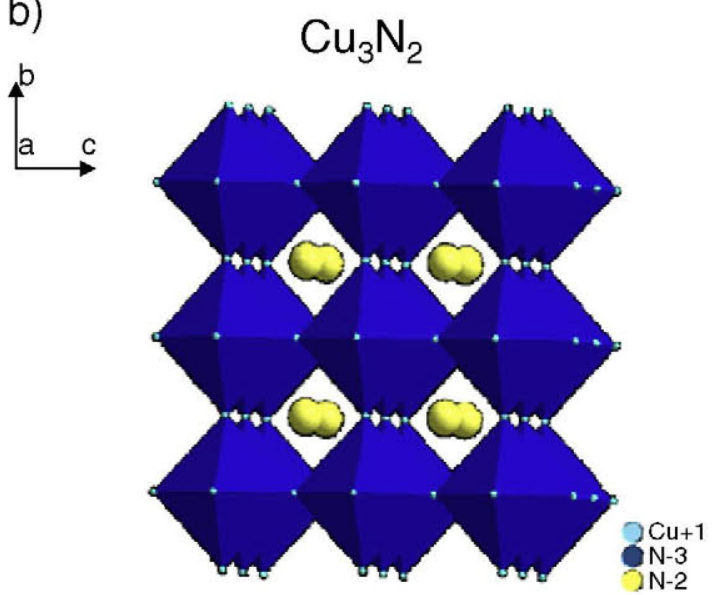

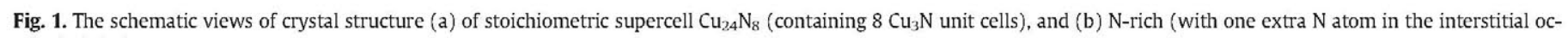
tahedral site).

Seebeck effect measurements have been carried out. Seebeck coefficient measurements have been applied to $\mathrm{Cu}_{3} \mathrm{~N}$ as a convenient alternative to Hall experiments $[23,28]$, that have not, so far, provided conclusive results. In fact, it has been claimed that Seebeck coefficient measurements often offer better reproducibility [29] than Hall Effect experiments. Moreover, in order to understand the experimental data, band structure and density of states (DOS) calculations have been also performed within the framework of the density functional theory $[7,30]$ as a function of nitrogen content for stoichiometric and $\mathrm{N}$-rich copper nitrides. Experimental and calculated data allow us to obtain a coherent picture of the effect of $\mathrm{N}$ content on the electrical and optical performances.

\section{Experimental and theoretical methods}

Copper nitride films with a thickness of $\sim 80 \mathrm{~nm}$ having a nitrogen concentration in the range from $26 \pm 2$ at.\% (referred from now on as stoichiometric $\mathrm{Cu}_{3} \mathrm{~N}$ ) to $33 \pm 2$ at.\% were deposited by DC-triode sputtering. The samples were deposited from a copper commercial target in the presence of an $\mathrm{Ar}+\mathrm{N}_{2}$ atmosphere on commercial $\mathrm{Si}$ (100) substrates at room temperature (RT) and DC bias of $2.0 \mathrm{kV}$. The total gas pressure $\left(\mathrm{Ar}+\mathrm{N}_{2}\right)$ and the target-substrate distance were kept constant at $8 \times 10^{-3}$ mbar and $8 \mathrm{~cm}$ respectively, whereas the nitrogen partial pressure was varied from $20 \%$ to $100 \%$. The plasma power was typically $60 \mathrm{~W}$ and a constant magnetic field of $10^{-4} \mathrm{~T}$ was applied for plasma confinement. X-ray diffraction data show that all films are polycrystalline and preferentially oriented along the (100) axes [10]. The elemental composition of the films was assessed by ion beam analysis techniques including Rutherford backscattering spectroscopy, non-Rutherford backscattering spectroscopy, and nuclear reaction analysis. More details about film deposition procedure, structural and elemental characterization are reported in Ref. [10].

Spectroscopic elliosometry (SE) spectra were measured as a function of temperature with a variable angle J. A. Woollam Co. ellipsometer model V-Vase in the wavelength range from 0.30 to $1.80 \mu \mathrm{m}$, at an incidence angle of $65^{\circ}$. The temperature range was selected to be between $100 \mathrm{~K}$ and room temperature (RT).

The electrical resistivity $(\rho)$ and the Seebeck coefficient $(S)$ were measured at RT by the Van der Pauw [31] and a differential method [32], respectively. The Seebeck coefficient was obtained by producing a temperature difference between the ends of the sample of 7-10 K by a heat source. Two point K-type thermocouples placed on the film surface measured the temperature difference, and two steel points, placed besides the thermocouples, were used to measure the generated thermovoltage. The whole system is thermally and electrically isolated from the surroundings. The Telkes criterion [33] about the sign of the Seebeck coefficient was accepted.

First principles calculations of the electronic structure for stoichiometric and $\mathrm{N}$-rich $\mathrm{Cu}_{3} \mathrm{~N}$ films were carried out within the density functional theory [30] using the generalized gradient approximation for the exchange and correlation to account for experimental data. The spin resolved calculations were performed with the SIESTA method [34] which uses a basis of numerical atomic orbitals and separable norm conserving pseudopotentials with partial core corrections. The standard double- $\varsigma$ basis with polarization orbitals was satisfactory found.

\section{Results and discussion}

Optical absorption data reported in a previous publication [26] for similar films show that stoichiometric samples behave as intrinsic semiconductors, whereas $\mathrm{N}$-rich films present an additional free carrier optical absorption (Drude) component which extends into the transparency range (below $\sim 1.3 \mathrm{eV}$ ) up to the middle infrared (IR). This IR band was assumed to be related to free carriers (electron or hole) associated to the introduction of in-gap levels by the extra nitrogen atoms. On the other hand, the high energy region of the spectra, corresponding to interband transitions, has been found to be almost the same for all samples, regardless of nitrogen contents. In the same work, an enhancement in the optical gap with increasing $\mathrm{N}$ content is also reported.

In order to test the above assumption the SE parameters, amplitude ratio $(\Psi)$ and the phase difference $(\Delta)$, were measured at different temperatures in the range from 100 to $300 \mathrm{~K}$ for the film with the highest $\mathrm{N}$ content. The data are depicted in Fig. 2 . In agreement with previously reported optical absorption data [26] all SE spectra present a high-absorbance region from around $1.3 \mathrm{eV}$ to $4.5 \mathrm{eV}$ that corresponds to the allowed interband transitions of the semiconductor plus an IR band, below $\sim 1.3 \mathrm{eV}$. Moreover, as shown in Fig. 2 the overall SE spectra are essentially independent of temperature indicating that the in-gap levels introduced by the $\mathrm{N}$ excess might be very close or inside the semiconductor bands.

For a better analysis of these experimental results, and to obtain a more complete picture of the electronic structure, density functional theory calculations were performed using the SIESTA code. For the stoichiometric $\mathrm{Cu}_{3} \mathrm{~N}$ compound the calculation yield an equilibrium lattice constant of $3.88 \AA$ ( $\mathrm{Cu}-\mathrm{N}$ bond length of $1.94 \AA$ ) which agrees well with the available experimental value for similar films [10] (3.86 $\AA$ ). The electronic calculations for $\mathrm{N}$-rich samples were done assuming a perfect $\mathrm{Cu}_{24} \mathrm{~N}_{8}$ super cell containing one extra $\mathrm{N}$ atom at the 


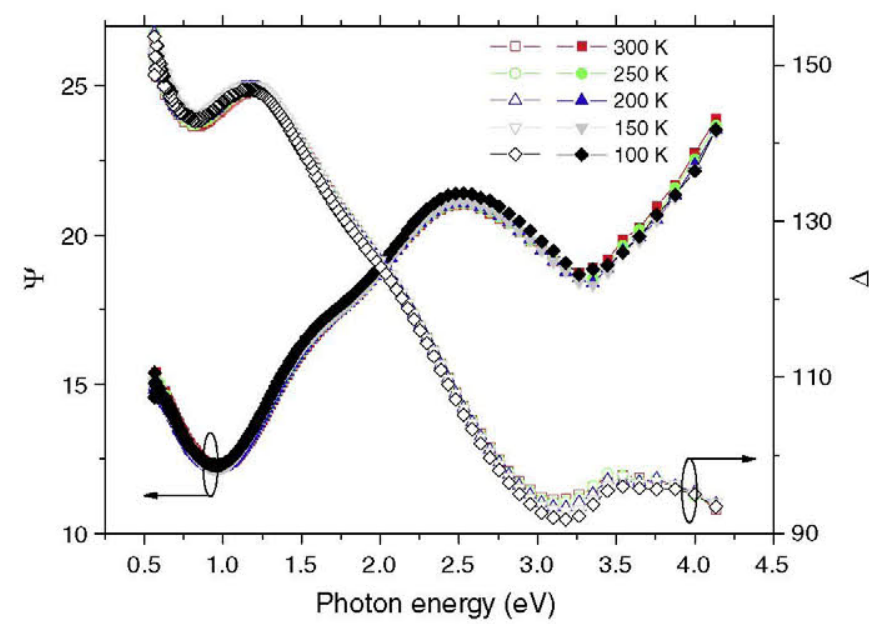

Fig. 2. SE spectra ( $\Psi$, full symbols and $\Delta$, open symbols) measured in the $0.30-1.80 \mu \mathrm{m}$ range at an incidence angle of $65^{\circ}$ at various temperatures in the range of $100-300 \mathrm{~K}$ for a $\mathrm{N}$ rich- $\mathrm{Cu}_{3} \mathrm{~N}$ sample with an atomic nitrogen percentage of $33 \pm 2$.

interstitial octahedral site (see Fig. 1(b)). Based on previous X-ray diffraction experimental data [10] for similar films (deposited under different conditions) which evidence insignificant changes (lower than $1 \%$ ) in the lattice constant with increasing $\mathrm{N}$ content, calculations were carried out by assuming the same lattice constant than for stoichiometric films [26]. Within this configuration the position of the nitrogen in the unit cell was relaxed until the residual forces were lower than $0.01 \mathrm{eV} / \mathrm{A}$. The extra $\mathrm{N}$ atom was found to be $0.08 \AA$ off the center of the unit cell along the (111) direction and bonded to three $\mathrm{Cu}$ atoms with a bond length of $2.16 \AA$.

The dispersion curves and DOS profiles for stoichiometric supercell $\mathrm{Cu}_{24} \mathrm{~N}_{8}$ (containing $8 \mathrm{Cu}_{3} \mathrm{~N}$ unit cells), and $\mathrm{N}$-rich (one extra $\mathrm{N}$ atom at one interstitial octahedral site) are depicted in Fig. 3(a), and (b), respectively. Data shown in Fig. 3(a) illustrates that stoichiometric samples behave as a semiconductor with an indirect absolute gap of $0.32 \mathrm{eV}$ and direct gaps of 1.09 and $0.87 \mathrm{eV}$ at the $\Gamma$ and $R$ points, respectively. This calculated indirect gap value is in between those $(0.13 \mathrm{eV}$ and $0.40 \mathrm{eV})$ predicted by different first principles approaches [7,24,35-37]. As shown in Fig. 3(b) the presence of extra $\mathrm{N}$ atoms in interstitial location does not substantially perturb the $\mathrm{Cu}_{3} \mathrm{~N}$ electronic structure as confirmed by the DOS profiles, but introduces partially-filled spin-resolved narrow bands of new electronic states in the semiconductor gap at the Fermi level $\left(E_{F}\right)$. Intra-band transitions within that intermediately band qualitatively account for the occurrence of the measured optical absorption in the near and medium IR for the N-rich films, as well as for the temperature independence of the SE spectra.

Additional information on the role of the free carriers and the electronic structure was derived from transport (Seebeck Effect and electrical resistivity) measurements. The dependence of the Seebeck coefficient, $S$, and of the electrical resistivity, $\rho$, on $\mathrm{N}$ atomic percentage ( $\mathrm{N}$ at.\%) is depicted in Fig. 4. All samples exhibit a negative $S$ coefficient which decreases from $\sim-5 \mu \mathrm{V} / \mathrm{K}$ to $-50 \mu \mathrm{V} / \mathrm{K}$ with rising nitrogen concentration from $26 \pm 2$ to $33 \pm 2$ at.\%. The negative sign of $S$ indicates that the majority of the carriers must be electrons, being consistent with previously reported Hall effect data $[23,28]$. The relatively small $S$ value for the stoichiometric film might indicate that electron and hole carrier densities are almost compensated, as expected for an intrinsic semiconductor. For $\mathrm{N}$-rich samples the absolute value of $S,|S|$, remarkably increases with rising nitrogen content, being up to one order of magnitude higher for the sample containing 33 at.\% of nitrogen than for the stoichiometric one. This strong dependence of the Seebeck coefficient on nitrogen content is, qualitatively, consistent with the SE data discussed in this paper and with the
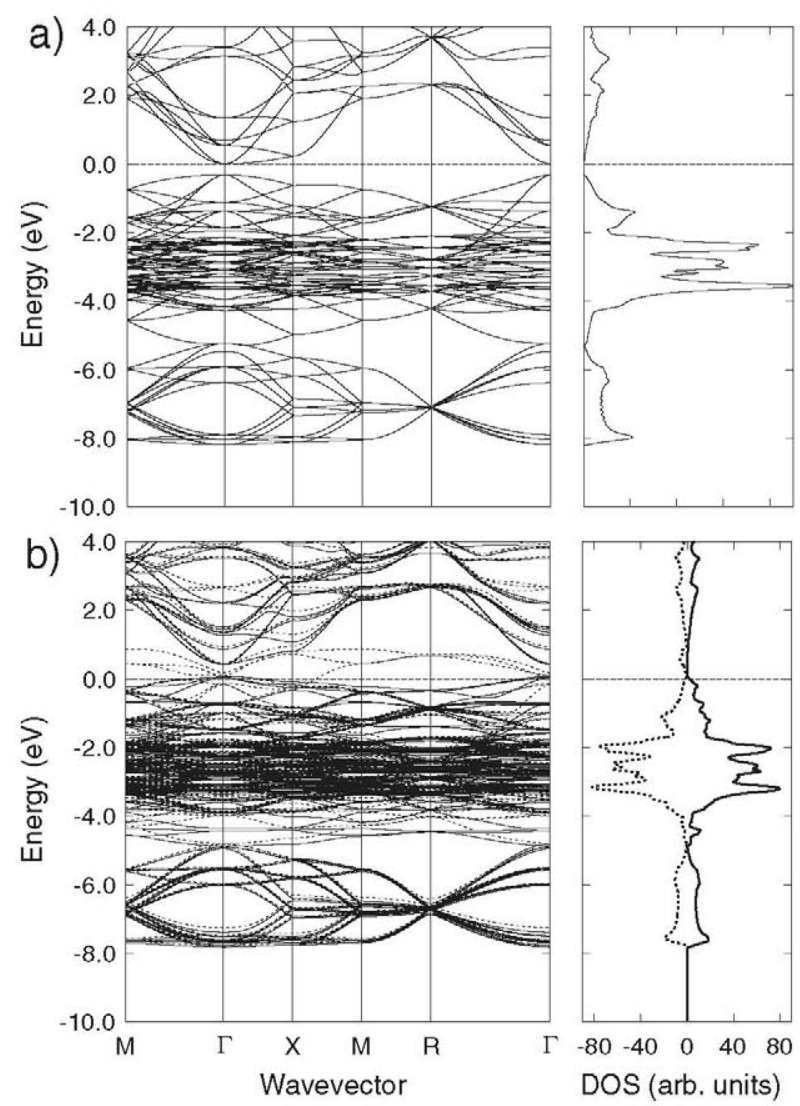

Fig. 3. Band structure and density of states for a stoichiometric supercell $\mathrm{Cu}_{24} \mathrm{~N}_{8}$ (a) and for a $\mathrm{Cu}_{24} \mathrm{~N}_{8}$ supercell with an extra $\mathrm{N}$ atom located in the center of the unit cell (b). In (b), continuous and broken lines stand for spin up and spin down states, respectively. The energy scale is relative to the Fermi level.

electronic structure picture obtained from density functional theory calculations. For certain transition metals $|S|$ has been found to strongly increase when doping them. Such an enhancement has been related to the narrowness of the $d$ bands and the abrupt increase of the resistivity near the Fermi level. A similar argument has been, recently, invoked to account for the high Seebeck coefficients measured in some oxides [38-40] such as cobaltates, rhodates and perovskites $\left(\mathrm{SrTiO}_{3}, \mathrm{KTaO}_{3}\right.$ and $\left.\mathrm{BaTiO}_{3}\right)$ when suitably doped with electrons and holes. In these cases, doping introduces a narrow additional band that is considered to be responsible for the high $|\mathrm{S}|$ values.

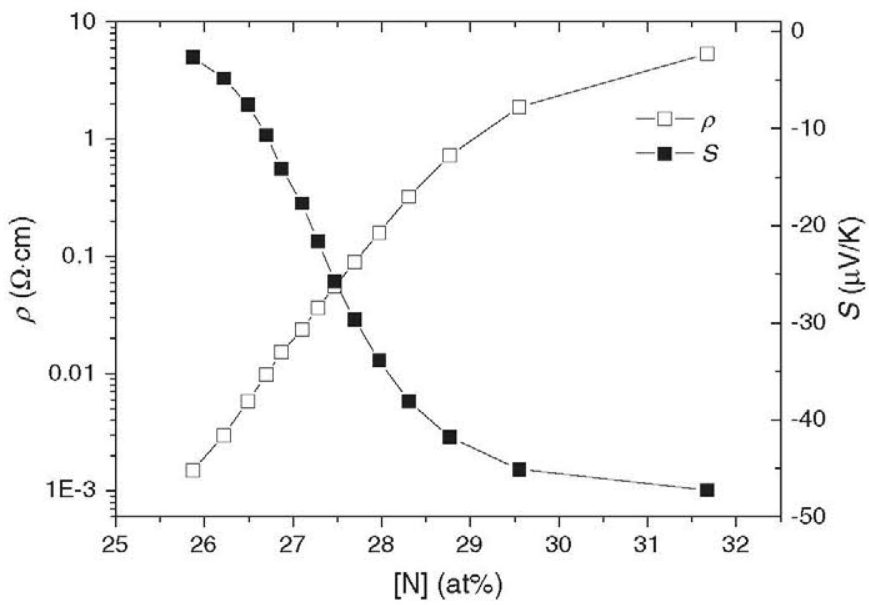

Fig. 4. Resistivity (open squares) and Seebeck coefficient (black squares) for copper nitride samples as a function of the atomic percentage of nitrogen. 
On these bases, we propose that the enhancement of $|S|$ value with increasing nitrogen concentration is mainly due to the occurrence of a narrow intermediate band and to the calculated abruptness of DOS profile at the Fermi level.

Concerning to the electrical resistivity, $\rho$, it is found to increase by four orders of magnitude, from $\sim 10^{-3} \Omega \mathrm{cm}$ to $10 \Omega \mathrm{cm}$, with rising nitrogen concentration [see Fig. 4]. The origin of the resistivity enhancement cannot be definitely explained in the previously described context. In principle, one could argue that the introduction of a quasi-metallic band at $E_{F}$ might reduce the resistivity of the films. However, this argument might not be valid if, as in our case, the electrons in that band have a very high effective mass (strong localized states) since their contribution to the conductivity has to be almost negligible. Another possible reason for the resistivity behavior is related to a reduction of the carrier concentrations due to the observed energy-gap broadening [26]. Moreover, it is, also, worthwhile to mention that the resistivity might be also influenced by the increases in the lattice disorder when introducing extra $\mathrm{N}$ atoms which should reduce the carriers' mobility.

\section{Summary and conclusion}

The electronic properties of $\mathrm{Cu}_{3} \mathrm{~N}$ films with an atomic percentage of nitrogen ranging from $26 \pm 2$ to $33 \pm 2$ at.\% have been investigated by combining optical (SE) and electrical (Seebeck effect and resistivity) measurements, together with first principle calculations, using the SIESTA method.

Experimental and calculated data illustrate that stoichiometric $\mathrm{Cu}_{3} \mathrm{~N}$ behaves as an intrinsic semiconductor whereas the electronic structure of $\mathrm{N}$-rich films contains the two bands (conduction and valence) corresponding to the stoichiometric $\mathrm{Cu}_{3} \mathrm{~N}$ plus a new partially filled narrow (quasi-metallic) band which is related to the $\mathrm{N}$ excess. The extra $\mathrm{N}$ lead to a remarkable increase in the absolute value of $S$ accompanied to a large enhancement in the electrical resistivity. According to Seebeck data the majority of the carriers must be electrons for all samples. The high negative $S$ values are consistent with the introduction of a narrow new band at the Fermi level and the abruptness of the density of states there. The origin of the resistivity behavior might be associated to the band gap widening and/or to the decrease of the carriers' mobility. Nevertheless, further research must be carried out in order (i.e. temperature dependent resistivity measurements) to completely understand the resistivity behavior.

In conclusion, we have shown how the introduction of extra $\mathrm{N}$ in stoichiometric $\mathrm{Cu}_{3} \mathrm{~N}$ strongly modifies the electrical properties of this compound.

\section{Acknowledgments}

The authors want to thank A. Âlvarez-Herrero for his helpful discussions and advices with the SE measurements. One of the authors (N.G.) acknowledge the M.C.Y.T. Grant No. FIS 2008-01431 for the financial support. Research by N. Gordillo was supported by a PICATA postdoctoral fellowship of the Moncloa Campus of International Excellence (UCM-UPM).

\section{References}

[1] D.M. Borsa, S. Grachev, D.O. Boerma, IEEE Trans. Magn. 38 (2002) 2709.

[2] M. Asano, K. Umeda, A. Tasaki, Jpn. J. Appl. Phys. 29 (1990) 1985.

[3] T. Maruyama, T. Morishita, Appl. Phys. Lett. 69 (1996) 890.

[4] T. Nosaka, M. Yoshikate, A. Akamoto, S. Ogawa, Y. Nakayama, Appl. Surf. Sci. 169-170 (2001) 358 .

[5] D.M. Borsa, S. Grachev, C. OPresura, D.o. Boerma, Appl. Phys. Lett. 80 (2002) 1823.

[6] X.D. Ma, D.I. Bazhanov, O. Fruchart, F. Yildiz, T. Tokoyama, M. Przybyiski, V.S. Stepanyuk, W. Hergert, M. Kirschner, Phys. Rev. Lett. 102 (2009) 205503.

[7] C. Navio, M.J. Capitan, J. Alvarez, F. Yndurain, R. Miranda, Phys. Rev. B 76 (2007) 085105.

[8] T. Törndahl, (2004) PhD thesis ISBN: 91-554-6081-X "Atomic layer deposition of copper, copper (I) oxide and copper (I) nitride on oxides substrates".

[9] C. Gallardo-Vega, W. de la Cruz, Appl. Surf. Sci. 252 (2006) 8001.

[10] N. Gordillo, R. Gonzalez-Arrabal, M.S. Martin-González, ]. Olivares, A. Rivera, F. Briones, F. Agulló-López, D. Boerma, J. Cryst. Growth 310 (2008) 4362.

[11] T. Maruyama, T. Morishita, J. Appl. Phys. 78 (1995) 4104.

[12] T. Nosaka, M. Yoshikate, A. Akamoto, S. Ogawa, Y. Nakayama, Thin Solid Films 348 (2001) 8.

[13] D.Y. Wang, N. Nakamine, Y. Hayashi, J. Vac. Sci. Technol. A 16 (1998) 2084.

[14] J.F. Pierson, Vacuum 66 (2002) 59.

[15] S. Terada, H. Tanaka, K. Kubota, J. Cryst. Growth 94 (1989) 567.

[16] K.J. Kim, K.H. Kim, J.H. Kang, J. Cryst. Growth 222 (2001) 767.

[17] A.L. Ji, R. Huang, C.R. Li, Y.Q. Wang, Z.X. Cao, J. Cryst. Growth 95 (2006) 79

[18] D.M. Borsa, D.O. Boerma, Appl. Surf. Sci. 548 (2004) 95.

[19] J. Wang, J.T. Chen, X.M. Yuan, Z.G. Wu, B.B. Miao, P.X. Yan, J. Cryst. Growth 286 (2006) 407

[20] J.M.D. Coey, P.A.I. Smith, J. Magn. Magn. Mater. 200 (1999) 405.

[21] I. Fernăndez, M.S. Martín-González, R. Gonzâlez-Arrabal, R. Âlvarez, F. Briones, J.L. Costa-Krämer, J. Magn. Magn. Mater. 320 (2008) 68.

[22] S.K. Shrestha, H. Timmers, K.S.A.S. Butcher, M. Wintrebert-Fouquet, Curr. Appl. Phys.A. 4 (2004) 237.

[23] G.H. Yue, P.X. Yan, J.Z. Liu, M.X. Wang, M. Li, X.M. Yuan, J. Appl. Phys. 98 (2005) 103506.

[24] M. Guadalupe Moreno-Armenta, A. Martinez-Ruiz, N. Takeuchi, Solid State Sci. 6 (2004) 9.

[25] Z.F. Hou, Solid State Sci. 10 (2008) 1651

[26] N. Gordillo, R. Gonzalez-Arrabal, A. Alvarez-Herrero, F. Agulló-López, J. Phys. D: Appl. Phys. 42 (2009) 165101.

[27] R. Gonzalez-Arrabal, N. Gordillo, M.S. Martín-González, R. Ruiz-Bustos, F. Agulló-López, J. Appl. Phys. 107 (2010) 103513.

[28] G.H. Yue, J.Z. Liu, M. Li, X.M. Yuan, P.X. Pan, J.L. Liu, Phys. Status Solidi A 202 (2005) 1987.

[29] J.R. Ares, I.J. Ferrer, C. Sánchez, Thin Solid Films 431-432 (2003) 511.

[30] W. Kohn, L.J. Sham, Phys. Rev. 140 (1965) A1133.

[31] L.J. Van der Pauw, Philips Tech. Rev. 20 (1958) 220.

[32] J.R. Ares, M. Leon, N.M. Arozamena, J. Sánchez-Paramo, P. Celis, I.J. Ferrer, C. Sánchez, J. Phys. Condens. Matter 10 (1998) 4281.

[33] M. Telkes, Am. Mineral. 35 (1950) 536.

[34] J.M. Soler, E. Artacho, J.D. Gale, A. Garcia, J. Junquera, P. Ordejôn, D. Sảnchez-Portal, J. Phys. Condens. Matter 14 (2002) 2745.

[35] U. Hahn, W. Weber, Phys. Rev. B 53 (1996) 12684.

[36] W. Yu, J.G. Zhao, C.Q. Jin, Phys. Rev. B 72 (2005) 214116

[37] A. Soon, L. Wong, M. Lee, M. Todorova, B. Delley, C. Stampfl, Surf. Sci. 601 (2007) 4775.

[38] D.J. Singh, Phys. Rev. B 61 (2000) 13397.

[39] G.B. Wilson-Short, D.J. Singh, M. Fornari, M. Suewattana, Phys. Rev. B 75 (2007) 035121.

[40] H. Usui, S. Shibata, K. Kuroki, Phys. Rev. B 81 (2010) 205121. 